Check for updates

Cite this: Chem. Sci., 2020, 11, 355

๑ All publication charges for this article have been paid for by the Royal Society of Chemistry

Received 16th September 2019 Accepted 14th November 2019

DOI: $10.1039 /$ c9sc04670d

rsc.li/chemical-science

\section{Ptychographic X-ray tomography reveals additive zoning in nanocomposite single crystals $\uparrow$}

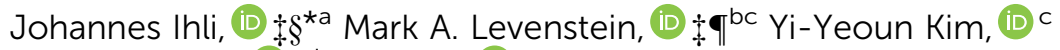 \\ Klaus Wakonig, (D) ad Yin Ning, (D) e Aikaterini Tatani, ${ }^{e}$ Alexander N. Kulak, (D) ${ }^{c}$ \\ David C. Green, (D) ${ }^{c}$ Mirko Holler, (D) ${ }^{\text {a }}$ Steven P. Armes (D) ${ }^{\mathrm{e}}$ and Fiona C. Meldrum (D) *c
}

\begin{abstract}
Single crystals containing nanoparticles represent a unique class of nanocomposites whose properties are defined by both their compositions and the structural organization of the dispersed phase in the crystalline host. Yet, there is still a poor understanding of the relationship between the synthesis conditions and the structures of these materials. Here ptychographic X-ray computed tomography is used to visualize the three-dimensional structures of two nanocomposite crystals - single crystals of calcite occluding diblock copolymer worms and vesicles. This provides unique information about the distribution of the copolymer nano-objects within entire, micron-sized crystals with nanometer spatial resolution and reveals how occlusion is governed by factors including the supersaturation and calcium concentration. Both nanocomposite crystals are seen to exhibit zoning effects that are governed by the solution composition and interactions of the additives with specific steps on the crystal surface. Additionally, the size and shape of the occluded vesicles varies according to their location within the crystal, and therefore the solution composition at the time of occlusion. This work contributes to our understanding of the factors that govern nanoparticle occlusion within crystalline materials, where this will ultimately inform the design of next generation nanocomposite materials with specific structure/property relationships.
\end{abstract}

\section{Introduction}

Nanocomposites are an exciting class of materials, where the ability to engineer compositions and structures at the nano- and meso-scales promises the ability to tailor electrical, optical, mechanical and catalytic properties, and to create multifunctionality. ${ }^{1-11}$ One strategy for creating these materials is by dispersing nanoparticles within a continuous host matrix. While this approach has been widely explored for polymers, ${ }^{\mathbf{1 , 1 2}-14}$ occlusion within inorganic matrices offers

\footnotetext{
${ }^{a}$ Paul Scherrer Institut, 5232 Villigen, Switzerland. E-mail: Johannes.Ihli@psi.ch ${ }^{b}$ School of Mechanical Engineering, University of Leeds, Leeds, LS2 9JT, UK ${ }^{c}$ School of Chemistry, University of Leeds, Leeds, LS2 9JT, UK. E-mail: F.Meldrum@ leeds.ac.uk

${ }^{d}$ Institute for Biomedical Engineering, ETHZürich, University of Zürich, 8093 Zürich, Switzerland

${ }^{e}$ Department of Chemistry, University of Sheffield, Sheffield, S3 7HF, UK

$\uparrow$ Electronic supplementary information (ESI) available: Materials and methods, further characterization of the crystals and polymers, supporting data for the ptychography analysis and two movies. See DOI: 10.1039/c9sc04670d

\$ Dr Johannes Ihli and Dr Mark Levenstein contributed equally to this work.

$\S$ Previous address: Department of Materials, University of Oxford, Oxford, OX2 6HT, UK.

I Present address: Department of Mechanical Science and Engineering, University of Illinois at Urbana-Champaign, 1206 West Green Street, Urbana, Illinois 61801, USA.
}

additional challenges, ${ }^{\mathbf{1 1 , 1 5 , 1 6}}$ where it is frequently difficult to control the microstructure and nanoparticles frequently accumulate at the grain boundaries in polycrystalline host matrices. ${ }^{17}$

Incorporation of pre-made nanoparticles within single crystal hosts can potentially overcome these problems. However, simple co-precipitation of salts such as $\mathrm{NaCl}$ or borax in aqueous solutions, ${ }^{18-20}$ and calcite $\left(\mathrm{CaCO}_{3}\right)^{21-23}$ in the presence of nanoparticles typically results in low levels of occlusion (under 1 wt\%). Superior occlusion can be achieved by functionalizing the nanoparticles with polymers that can act as steric stabilizers and promote binding to the surface of the host crystal. This approach has been demonstrated for the occlusion of nanoparticles including magnetite $\left(\mathrm{Fe}_{3} \mathrm{O}_{4}\right)$, gold and organic nano-objects in calcite and $\mathrm{ZnO}^{24-28}$ Nanoparticle aggregation is less significant in organic solvents, such that successful occlusion has been achieved of Pt nanoparticles in $\mathrm{MOFs}^{29}$ and $\mathrm{PbS}$ particles in methylammonium lead iodide perovskites. ${ }^{4,30}$

In all of these cases, the properties of these single crystal nanocomposites are dependent not only on their compositions, but also on their 3D structures. In principle, this enables control over material properties, where the size, shape, loading and distribution of the species occluded within the crystal matrix can all be varied. However, achieving this degree of control remains challenging because (i) our understanding of the 
fundamental mechanisms governing occlusion remains limited and (ii) there are inherent technical difficulties associated with visualising the 3D spatial organisation of the nanocomposite over extended volumes at an appropriate resolution. Techniques such as confocal fluorescence microscopy or optical microscopy can provide information about the average distribution of fluorescent or colored additives within a crystal, but the resolution is frequently insufficient to identify the locations of individual nano-sized or molecular additives. ${ }^{31,32}$ Serial sectioning and successive electron microscopy of samples can be carried out to build a tomographic image, but is both destructive and prone to artefacts. ${ }^{23}$ While significant advances have been made in electron tomography, ${ }^{33,34}$ this technique remains limited in the size of sample that can be studied, such that it is difficult to correlate the locations of the occlusions with the morphology of the host crystal.

Here, we use a model system comprising calcite single crystals incorporating two contrasting additives - diblock copolymer worms, and silica-loaded diblock copolymer vesicles - to investigate the parameters controlling the distribution of these species within a crystal host. Cryo-ptychographic X-ray computed tomography (PXCT) was used to visualize the internal structures of entire crystals. This non-destructive coherent diffraction imaging technique provides $3 \mathrm{D}$ quantitative electron density maps of extended specimens, ${ }^{35-39}$ and therefore enables the precise locations of the occluded nanoparticles to be correlated with the crystal morphology and structure. Our results not only reveal two forms of nanoparticle zoning, but also show that the size and shape of the additives incorporated vary according to their location within the crystal. This work provides important new insights into the influence of the crystal growth conditions and crystal/additive interactions on additive occlusion, where this will ultimately enable the design of nanocomposite materials with specific structure/property relationships.

\section{Results and discussion}

\section{Polymer nano-objects and nanocomposite synthesis}

Poly(methacrylic acid $)_{x}$-poly(benzyl methacrylate) $)_{y}\left(\mathrm{PMAA}_{x}{ }^{-}\right.$ PBzMA $_{y}$ ) diblock copolymer worms and vesicles were selected for this study because they offer strongly contrasting morphologies and sizes. These nanoparticles were prepared using polymerisation-induced self-assembly and are known to occlude within calcite due to the anionic surface character of the poly(methacrylic acid) stabiliser chains (Fig. 1a and b)..$^{25,40}$ For consistency with previous studies, ${ }^{40}$ silica nanoparticles were incorporated within the vesicles during their synthesis. The $\mathrm{PMAA}_{69}-\mathrm{PBzMA}_{200}$ vesicles (subscripts refer to the mean degree of polymerisation of each block) possess an intensityaverage diameter of $232 \mathrm{~nm}$ but ranged in size from 100 to $600 \mathrm{~nm}$, as determined by dynamic light scattering. ${ }^{40}$ The PMAA $_{71}-$ PBzMA $_{150}$ worms possessed average diameters of $\approx 30 \mathrm{~nm}$ and lengths of $>1 \mu \mathrm{m}$ as determined by transmission electron microscopy (TEM) (Fig. S1†). ${ }^{25}$

Nanocomposites were precipitated using the ammonia diffusion method (ADM), ${ }^{41}$ where a calcium chloride solution containing the diblock copolymer nano-objects is exposed to
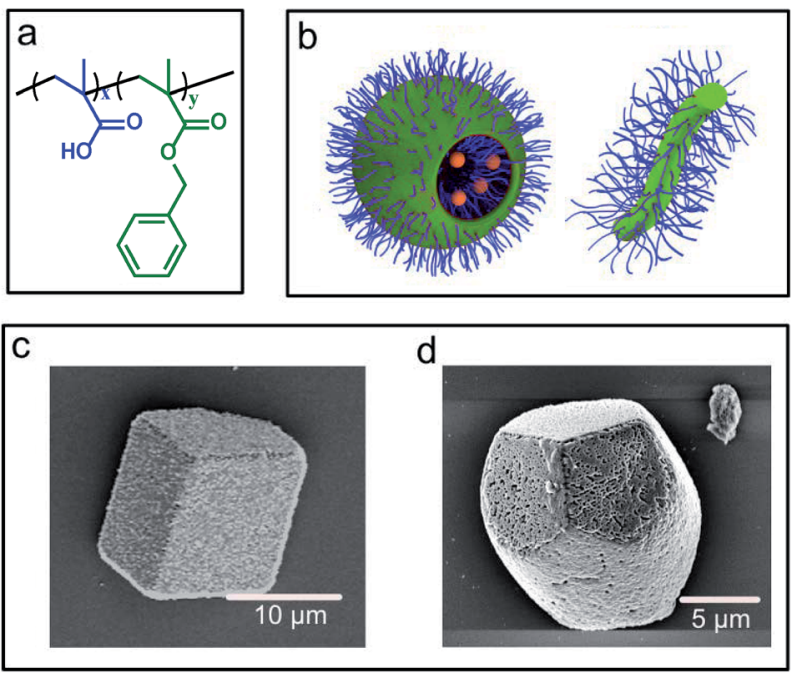

Fig. 1 (a) Generic chemical structure of the $\mathrm{PMAA}_{x}-\mathrm{PBzMA}_{y}$ diblock copolymer nano-objects used in the study. (b) Schematic representation of $\mathrm{PMAA}_{69}-\mathrm{PBz}_{2} \mathrm{~A}_{200}$ vesicles and $\mathrm{PMAA}_{71}-\mathrm{PBzMA}_{150}$ worms. (c) SEM images of vesicle/calcite and a (d) worm/calcite composite single crystals.

gaseous carbon dioxide and ammonia. This results in the formation of calcite $\left(\mathrm{CaCO}_{3}\right)$ single crystals occluding the polymer nano-objects, where the polymorph was confirmed using Raman microscopy (Fig. S1†). The two types of nanocomposite crystals exhibited distinct morphologies (Fig. 1c and d). The vesicle/calcite crystals retained the rhombohedral morphology of pure calcite, while the worm/calcite crystals were elongated along the $c$-axis, possessed rough surfaces, and their apexes were each capped by three smooth $\{104\}$ faces. These crystal morphologies reflect the differing degree of interaction of the anionic carboxylate groups on the copolymer particles with the growing crystals. Occlusion levels of $\approx 15-20 \mathrm{wt} \%$ for the worms ${ }^{25}$ and $\approx 25 \mathrm{wt} \%$ for the vesicles ${ }^{40}$ were estimated using thermogravimetric analysis (TGA) (Fig. S2 $\dagger$ ).

\section{Ptychographic tomography of nanocomposites}

The spatial organization of the worms and vesicles within individual calcite crystals were determined using cryoptychographic X-ray computed tomography (cryo-PXCT) at $-180{ }^{\circ} \mathrm{C}^{35,37,42}$ These measurements were carried out by randomly selecting crystals that were $10-50 \mu \mathrm{m}$ in diameter, and mounting them on custom-made tomography pins (Fig. S3 $†$ ). The resulting quantitative electron density tomograms possess a sample-average spatial resolution of $62 \mathrm{~nm}$ for the worm/calcite crystal and $56 \mathrm{~nm}$ for the vesicle/calcite crystal (Fig. S4 $\dagger$ ). The resolution is therefore more than sufficient to characterise the morphologies of the vesicles. For any analysis where the resolution is insufficient (e.g. of vesicle morphologies) the analysis was restricted to larger -resolvable- vesicles. It is noted that the area of the nanocomposite crystals in closest proximity to the tomography pin is omitted from the tomogram, giving an apparent truncation. For further method and samplesynthesis related information please refer to the accompanying ESI. $\dagger$ 


\section{Ptychographic tomography of a vesicle/calcite nanocomposite}

Fig. 2a shows a volume rendering, and two orthogonal slices through the electron density tomogram of a calcite crystal containing vesicles. The rhombohedral sample morphology that is apparent in the volume rendering is fully consistent with the SEM image shown in Fig. 1c. The orthoslices through the crystal reveal the locations of the vesicles in the calcite host and show that they are distributed in a non-uniform manner (Fig. 2a). The corresponding electron density histogram is shown in Fig. $2 \mathrm{~b}$, on which the theoretical electron densities of the two components of the nanocomposite - calcite $\left(0.82 \mathrm{eA}^{-3}\right)$ and silica-loaded copolymer vesicle $\left(0.36 \mathrm{eA}^{-3}\right)$ - are marked. As the physical density and composition of calcite and vesicles is known, their implied electron densities can be used to determine both the local, and the total volume fraction (or mass fraction) of the occluded vesicles. That this can be achieved without having to fully resolve the polymer nano-objects is due to the samples simple 2-component nature and the quantitative aspect of PXCT. Based on this analysis, the PXCT data suggest that this calcite crystal occludes $\approx 17 \mathrm{wt} \%$ vesicles, which is in reasonable agreement with the TGA data. The deviation between these values may arise from crystal-to-crystal variations.

Orthoslices through the tomogram clearly show that the crystal comprises a vesicle-poor ( $<10 \mathrm{wt} \%$ vesicles) rhombohedral core of diameter $\approx 12 \mu \mathrm{m}$, surrounded by $a \approx 12 \mu \mathrm{m}$ thick vesicle-rich ( $\approx 23 \mathrm{wt} \%$ vesicles) zone. There is then a second, vesicle-poor zone, which increases slightly in vesicle content in the outermost layer ( $\approx 15 \mathrm{wt} \%$ vesicles, Movie $\mathrm{S} 1 \dagger)$. These radial variations in vesicle occlusion efficacy are illustrated in Fig. 2c, which provides a radially-averaged electron density profile, expressed in terms of the distance from the proposed nucleation center of the crystal.

More information about the occluded vesicles was then obtained by applying a segmentation threshold to separate the calcite host from the vesicles, and then characterizing the diameter and sphericity $\Psi$ of individual vesicles. A cut-off value of 50 vol\% vesicle (or $<0.6 \mathrm{eA}^{-3}$ ) was applied and the segmentation
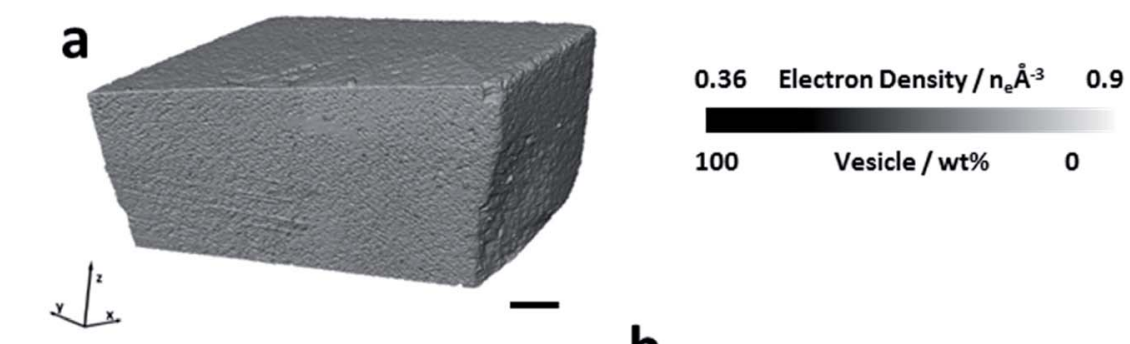

b
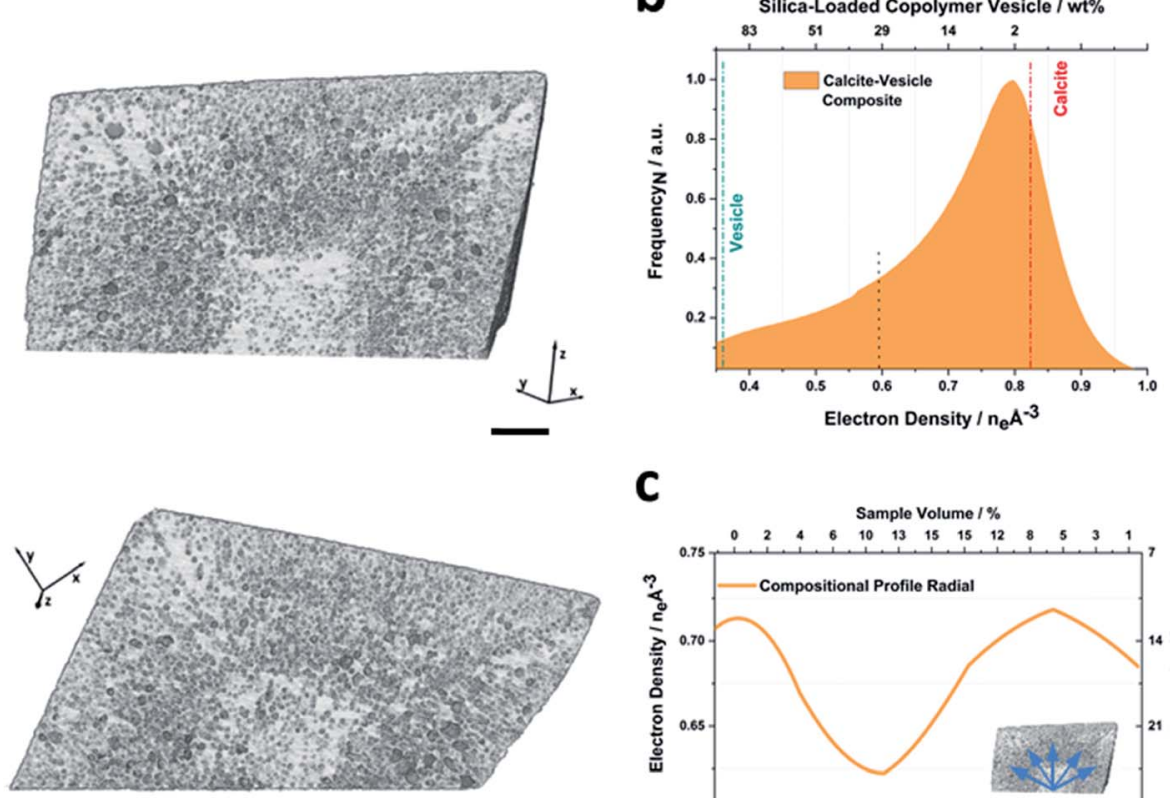

C

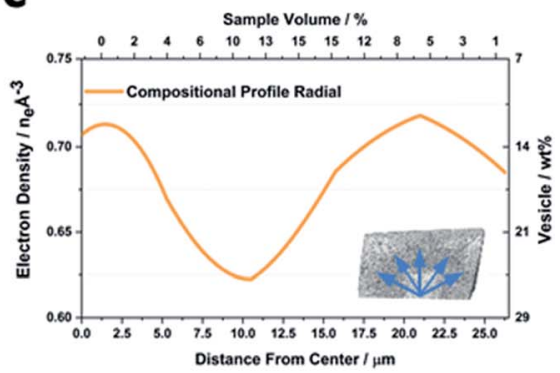

Fig. 2 Ptychographic X-ray computed tomography of a calcite nanocomposite crystal occluding diblock copolymer vesicles. (a) Volume rendering of and orthoslices through the electron density tomogram of a calcite crystal occluding silica-loaded copolymer vesicles (PMAA $_{69}-$ $\left.\mathrm{Bz}_{\mathrm{B}} \mathrm{A}_{300}\right)$. Both orthoslices are shown with a single color scale ranging from black to white, where this is representative of the electron density value or the approximate weight percent of vesicles. (b) Corresponding histogram, where the electron densities of the reference components and the applied segmentation thresholds are marked. (c) Radial distribution profiles showing the volume averaged compositional variation of the nanocomposite as a function of distance from the crystal center (blue arrows). The scale bars are $5 \mu \mathrm{m}$, the voxel size is (16.6 nm) ${ }^{3}$ and measurements were conducted under cryogenic conditions. 
was refined through the application of morphological operators to ensure that entire vesicles were included in the analysis. The segmented volume rendering and orthoslices provided in Fig. 3a show the locations of individual vesicles within the crystal matrix. The average inter-vesicle spacing was determined to be $\approx 300 \mathrm{~nm}$ (Fig. S5 $\dagger$ ), and on average the vesicles retain their solution sphericity $(\Psi \approx 0.98)$ and mean diameters of $210 \mathrm{~nm}$ upon occlusion. The occluded vesicle size distribution is an almost perfect match to that in solution (Fig. 3b), which demonstrates that the vesicles experience minimal aggregation or deformation during occlusion. A bivariate histogram of vesicle size vs. sphericity reveals that if significant shape changes do occur, they principally affect larger vesicles (Fig. 3b). Finally, given the rhombohedral symmetry of calcite and the corresponding morphology of the composite crystal we investigated the radial dependency of vesicle size and sphericity from the nucleation centre, i.e. the vesicle-poor core, to the composite crystal exterior (Fig. 3c). Evident from these distributions is that, although larger
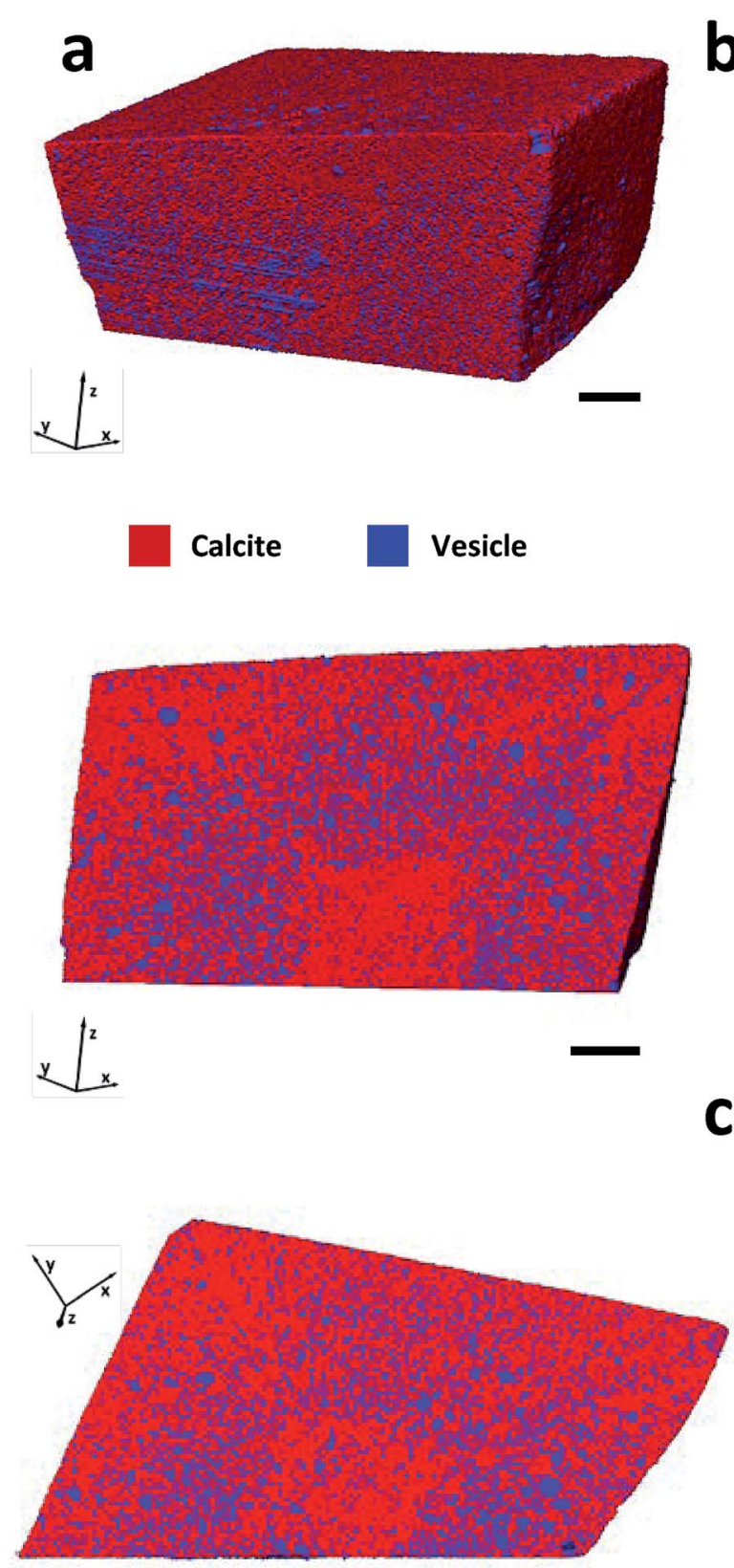

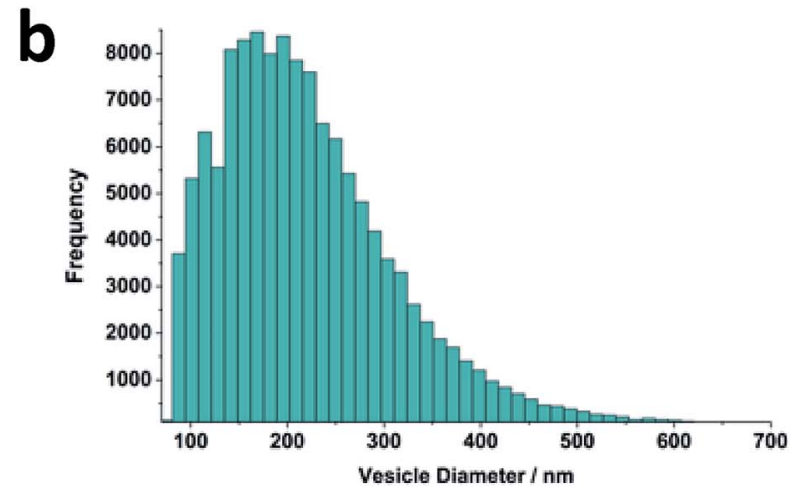

0 Frequency 100

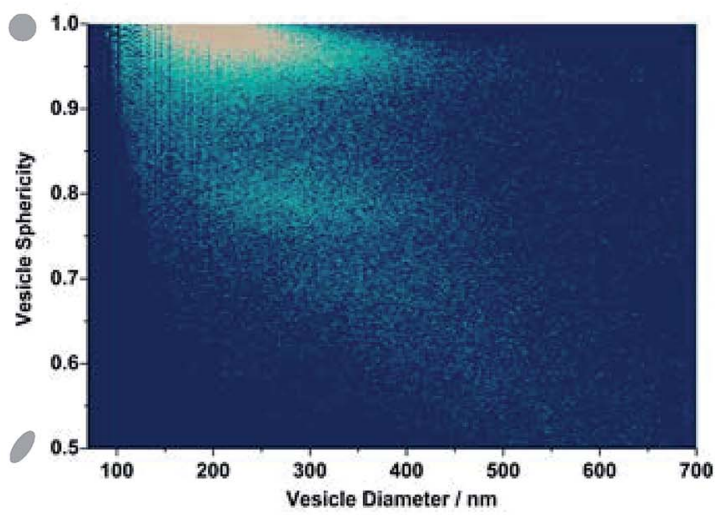

C
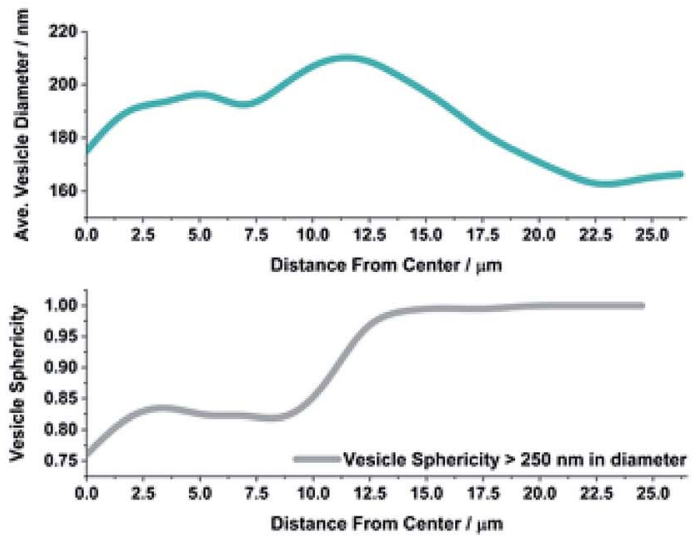

Fig. 3 Threshold segmented tomography of a calcite nanocomposite crystal occluding diblock copolymer vesicles. (a) Volume rendering of and orthoslices through the threshold-segmented tomogram. The segmentation threshold used to separate the calcite host (red) from the dispersed silica-loaded vesicles (blue) was $\sim 0.6 \mathrm{eA}^{-3}$. (b) Vesicle size distribution and bivariate histogram showing the number of vesicles as a function of diameter and sphericity. (c) Radial distribution profiles showing the variations in the volume averaged vesicle diameter and sphericity of larger vesicles as a function of the distance from the composite center. Scale bars are $5 \mu \mathrm{m}$ and the voxel size is $(16.6 \mathrm{~nm}){ }^{3}$ 
vesicles are preferentially occluded in zones of higher occlusion density (i.e. the zone immediately surrounding the low vesicle density core), the vesicle sphericity increases from the core of the nanocomposite crystal to its outer surface.

\section{Ptychographic tomography of a worm/calcite nanocomposite}

The volume rendering of the electron density tomogram of a worm/calcite crystal and the corresponding orthoslices are shown in Fig. 4a. The elongated morphology is in excellent
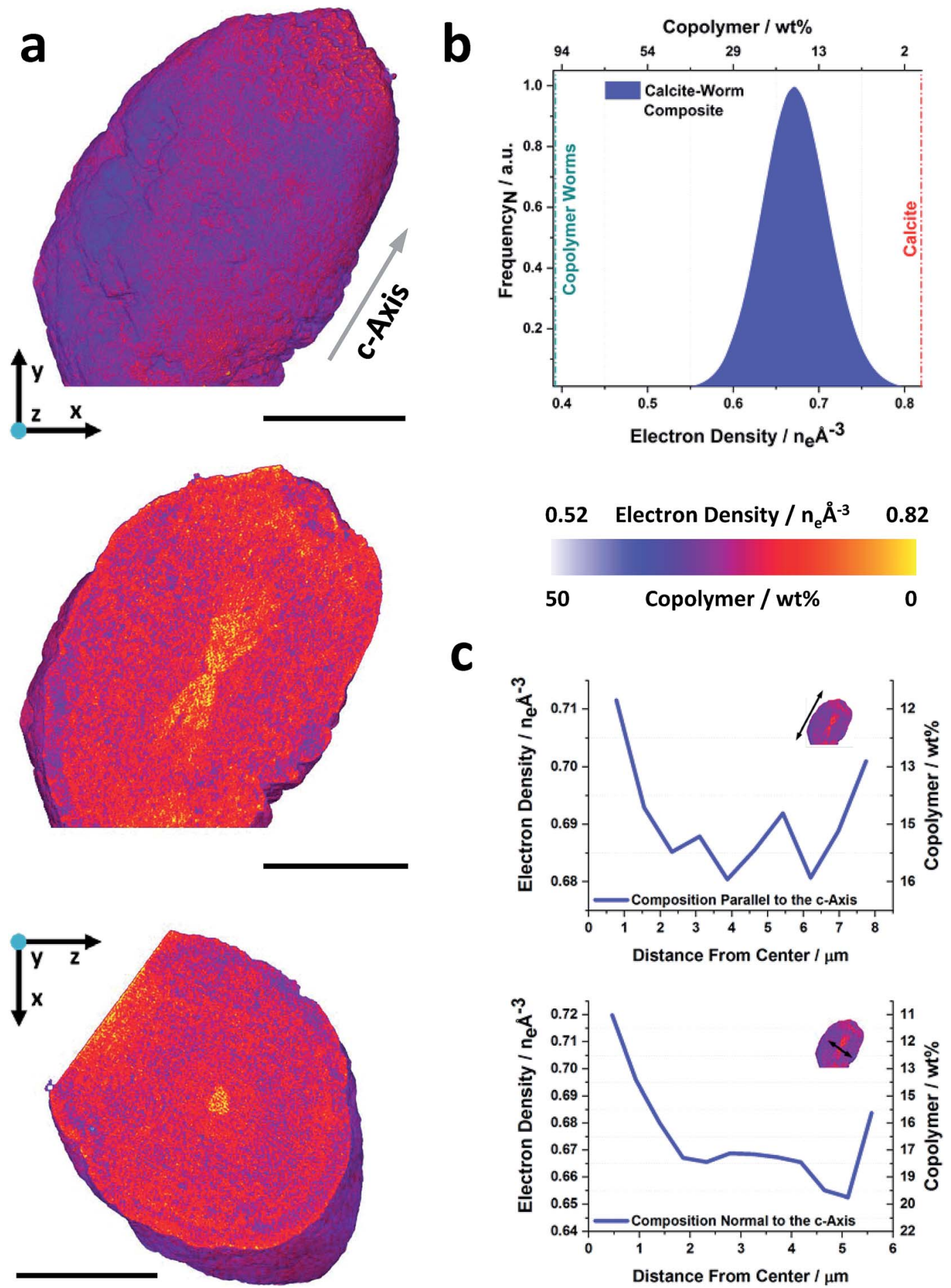

Fig. 4 Ptychographic X-ray computed tomography of a calcite nanocomposite crystal occluding diblock copolymer worms. (a) Volume rendering, and orthoslices through the electron density tomogram of a calcite crystal occluding copolymer worms ( $\mathrm{PMAA}_{70}-\mathrm{BzMA}_{150}$ ). Common to both orthoslices is a single color scale ranging from white to yellow, where this is representative of the electron density value or weight percent of copolymer. (b) Electron density histogram, electron densities of the reference components are indicated. (c) Radial distribution profiles showing the compositional variation parallel and normal to the crystallographic $c$-axis as a function of distance from the composite center. The scale bars are $5 \mu \mathrm{m}$, the voxel size is $(38.8 \mathrm{~nm})^{3}$ and the measurements were conducted under cryogenic conditions. 
agreement with the SEM image and the average electron density suggests a composition of $\approx 16 \mathrm{wt} \%$ polymer worms (Fig. $4 \mathrm{~b}$ ), which again agrees well with the TGA analysis (18 wt\%). However, in contrast to the vesicle/calcite crystal, the orthoslices reveal that this crystal possesses both a radial and directional variation in occlusion. A worm-poor zone $(\approx 10 \mathrm{wt} \%$ worms) is evident, which takes the form of an hourglass whose axis runs parallel to the crystallographic $c$-axis. This hourglass shape is a common zoning effect in single crystals, where the symmetry is consistent with the nucleation point lying at the centre of the hourglass.

This zone is surrounded by a worm-rich zone $(\approx 18 \mathrm{wt} \%$ worms), followed by an outer worm-poor layer $(\approx 12 \mathrm{wt} \%$ worms) which follows the form of the hourglass core. The occlusion pattern is therefore more complex than that of the vesicle/calcite crystals, as is also shown in the averaged electron density line profiles shown in Fig. 4c. These data confirm that higher occlusion occurs in the equatorial zone and that occlusion is lower in the direction of elongation (the $c$-axis) (Movie $\mathrm{S} 2 \dagger)$. No further segmentation of these data was carried out as the spatial resolution was insufficient to fully resolve the worms within the host crystal.

\section{Origin of zoning effects}

Two forms of zoning are observed in these nanocomposite crystals. The first derives from selective interaction of the polymer nano-objects with the growth steps on the calcite surface and is observed in the worm/calcite crystals only. The morphology of these crystals - which are elongated along the $c$ axis and exhibit rough faces in the equatorial zone - derives from preferential binding of these additives to the acute step edges. ${ }^{43}$ This interaction is also apparent in the zoning pattern within the crystal, where the worms are again concentrated in the equatorial zone. This form of zoning is termed intra-sectoral zoning and is recognized for a range of ions including $\mathrm{Mg}^{2+}$, $\mathrm{Mn}^{2+}, \mathrm{Sr}^{2+}$ and $\mathrm{SO}_{4}{ }^{2-}$ (ref. 44-46) and also dye molecules in calcite. ${ }^{43}$

The second zoning effect is observed as a radial variation in the number density of occluded species. This appears as a lowocclusion core, surrounded by a higher occlusion zone. The occlusion density is principally determined by two factors, (i) the conformation of the anionic PMAA stabiliser chains, which is governed by the $\mathrm{Ca}^{2+} /$ carboxylate molar ratio and (ii) the growth rate. Considering the first of these factors, greater conformational freedom of the PMAA chains is associated with superior occlusion. The behaviour of weak polyelectrolyte brushes (such as PMAA) in the presence of monovalent and divalent ions has been well-studied. ${ }^{4-49} \mathrm{Ca}^{2+}$ ions interact strongly with anionic carboxylate groups, leading to more compact chain conformations and promoting bridging between neighbouring carboxylate groups. This behaviour is strongly dependent on the solution conditions, where subtle changes in the $\mathrm{Ca}^{2+}$ concentration can lead to sudden contraction or expansion of the PMAA stabiliser chains.

For a given copolymer concentration, efficient nanoparticle occlusion is thus determined by the calcium ion concentration, with higher concentrations leading to more compact PMAA chains and poor occlusion. The radial variation in occlusion observed in the calcite/vesicle crystals can therefore be attributed to the reduction in calcium ions that occurs during crystallisation. There is a relatively high $\mathrm{Ca}^{2+}$ concentration at the outset of crystallization which limits occlusion. The chains then recover their conformational freedom as the $\mathrm{Ca}^{2+}$ concentration decreases, leading to increased occlusion. We recently reported a similar effect when systematically varying the length of the anionic stabiliser chains for the occlusion of anionic crosslinked vesicles within calcite. ${ }^{50}$ As the conformation freedom is greater for longer stabilizer chains, vesicles stabilized by short chains were only incorporated near the outer surface of calcite crystals (when the $\mathrm{Ca}^{2+}$ concentration was low), while longer stabiliser chains led to extensive vesicle occlusion.

Finally, that both the worm/calcite and vesicle/calcite crystals exhibit a further decrease in occlusion close to the surface of the crystals can be attributed to the slowed crystal growth as crystallization terminates. For most additives, occlusion is limited by their residence time on the crystal surface. Occlusion is therefore favored at higher supersaturation, when an additive is rapidly engulfed before it has time to desorb. ${ }^{51}$

\section{Influence of occlusion process on incorporated additives}

Our data also provide a unique opportunity to evaluate the effect of occlusion on the polymer nano-objects themselves. The average sphericity of the incorporated vesicles decreases significantly with an increase in the vesicle size. While the smaller vesicles retain near perfect sphericity, larger vesicles $>250 \mathrm{~nm}$ in diameter can undergo significant shape changes and elongate during occlusion (Fig. $3 \mathrm{~b}$ and c). This can be attributed to the higher curvature of the smaller vesicles, which makes them harder to deform. However, the limitations in spatial resolution need to be taken into consideration when defining the sphericity of the smallest vesicles. Changes in shape have also been observed previously when polymer micelles were occluded in calcite, where the soft micelle flattens as it adsorbs to the crystal surface. ${ }^{24,52}$

\section{Conclusions}

The occlusion of additives ranging from small molecules, ${ }^{7,32,53,54}$ to polymers, ${ }^{34,55,56}$ to organic and inorganic nanoparticles, ${ }^{4,5,8,18,22,24,26,28}$ in single crystals provides a unique strategy for creating new materials with tailor-made properties. However, to fully profit from this approach, it is necessary to control the composition and structure of these nanocomposites. This can only be achieved by determining how occlusion is governed by the synthesis conditions and the interplay between the additive chemistry and the host crystal. Ideally, this is achieved by characterizing the 3D structures of such nanocomposite crystals. Here, we have demonstrated that this can be achieved using cryo-PXCT, which enabled us to nondestructively map the 3D distribution of copolymer worms and vesicles occluded within entire micron-sized calcite crystals. Our results reveal a non-uniform distribution of the 
nanoparticles that is governed by the synthesis conditions and the crystallography of the host crystal, and a local variation in the vesicle loading of up to $100 \mathrm{wt} \%$. This new insight into occlusion mechanisms will ultimately enable us to develop occlusion strategies to design and fabricate new nanocomposite materials with improved optical, structural and mechanical properties, where analyses of structure/property relationships are often made based on an assumption of a spatially uniform structure.

\section{Author contributions}

The manuscript was written through contributions of all authors. All authors have given approval to the final version of the manuscript.

\section{Funding sources}

The work of JI and WK is supported by funding from the Swiss National Science Foundation (SNF), grants 200021, 179886 and 153556. MAL is supported by a Leeds International Research Scholarship. We thank the Engineering and Physical Sciences Research Council (EPSRC) for funding under grant EP/P005233/ 1 (DCG and FCM) and EP/P005241/1 (YN and SPA). We also acknowledge an EPSRC Programme Grant (EP/R018820/1) which funds the Crystallization in the Real World consortium (FCM and YYK). Instrumentation was supported by SNF (R'EQUIP, 145056, "OMNY") and the Competence Centre for Materials Science and Technology (CCMX) of the ETH-Board, Switzerland.

\section{Conflicts of interest}

There are no conflicts to declare.

\section{Acknowledgements}

We thank X. Donath and Andreas Menzel for technical support at the cSAXS beamline. We would further like to thank Jesse N. Clark, Xiaowen Shi and David A. Shapiro for help with the initial PXCT experiments.

\section{References}

1 S. J. Zhang, C. I. Pelligra, X. D. Feng and C. O. Osuji, Directed Assembly of Hybrid Nanomaterials and Nanocomposites, Adv. Mater., 2018, 30(18), 23.

2 Y. N. Hao, X. H. Wang, K. Bi, J. M. Zhang, Y. H. Huang, L. W. Wu, P. Y. Zhao, K. Xu, M. Lei and L. T. Li, Significantly enhanced energy storage performance promoted by ultimate sized ferroelectric BaTiO3 fillers in nanocomposite films, Nano Energy, 2017, 31, 49-56.

3 A. P. Chen, Q. Su, H. Han, E. Enriquez and Q. X. Jia, Metal Oxide Nanocomposites: A Perspective from Strain, Defect, and Interface, Adv. Mater., 2019, 31(4), 1803241.

4 Z. Ning, X. Gong, R. Comin, G. Walters, F. Fan, O. Voznyy, E. Yassitepe, A. Buin, S. Hoogland and E. H. Sargent,
Quantum-dot-in-perovskite solids, Nature, 2015, 523, 324328.

5 D. C. Green, M. A. Holden, M. A. Levenstein, S. H. Zhang, B. R. G. Johnson, J. G. de Pablo, A. Ward, S. W. Botchway and F. C. Meldrum, Controlling the fluorescence and room-temperature phosphorescence behaviour of carbon nanodots with inorganic crystalline nanocomposites, Nat. Commun., 2019, 10, 206.

6 C.-W. Nan and Q. Jia, Obtaining ultimate functionalities in nanocomposites: Design, control, and fabrication, MRS Bull., 2015, 40(9), 719-724.

7 Y. Y. Kim, J. D. Carloni, B. Demarchi, D. Sparks, D. G. Reid, M. E. Kunitake, C. C. Tang, M. J. Duer, C. L. Freeman, B. Pokroy, K. Penkman, J. H. Harding, L. A. Estroff, S. P. Baker and F. C. Meldrum, Tuning hardness in calcite by incorporation of amino acids, Nat. Mater., 2016, 15(8), 903-912.

8 A. N. Kulak, M. Semsarilar, Y.-Y. Kim, J. Ihli, L. A. Fielding, O. Cespedes, S. P. Armes and F. C. Meldrum, One-pot synthesis of an inorganic heterostructure: uniform occlusion of magnetite nanoparticles within calcite single crystals, Chem. Sci., 2014, 5, 738-743.

9 R. Marschall, Semiconductor Composites: Strategies for Enhancing Charge Carrier Separation to Improve Photocatalytic Activity, Adv. Funct. Mater., 2014, 24(17), 2421.

10 S. Tamulevicius, S. Meskinis, T. Tamulevicius and H. G. Rubahn, Diamond like carbon nanocomposites with embedded metallic nanoparticles, Rep. Prog. Phys., 2018, 81(2), 024501.

11 Z. H. Chen, X. Wang, Y. J. Qi, S. Yang, J. Soares, B. A. Apgar, R. Gao, R. J. Xu, Y. Lee, X. Zhang, J. Yao and L. W. Martin, Self-Assembled, Nanostructured, Tunable Metamaterials via Spinodal Decomposition, ACS Nano, 2016, 10(11), 10237-10244.

12 M. Kotal and A. K. Bhowmick, Polymer nanocomposites from modified clays: Recent advances and challenges, Prog. Polym. Sci., 2015, 51, 127-187.

13 K. I. Winey and R. A. Vaia, Polymer nanocomposites, MRS Bull., 2007, 32(4), 314-319.

14 B. Sarkar and P. Alexandridis, Block copolymer-nanoparticle composites: Structure, functional properties, and processing, Prog. Polym. Sci., 2015, 40, 33-62.

15 N. Bahlawane, K. Kohse-Hoinghaus, T. Weimann, P. Hinze, S. Rohe and M. Baumer, Rational Design of Functional Oxide Thin Films with Embedded Magnetic or Plasmonic Metallic Nanoparticles, Angew. Chem., Int. Ed., 2011, 50(42), 99579960.

16 K. Biswas, J. Q. He, Q. C. Zhang, G. Y. Wang, C. Uher, V. P. Dravid and M. G. Kanatzidis, Strained endotaxial nanostructures with high thermoelectric figure of merit, Nat. Chem., 2011, 3(2), 160-166.

17 Y. Liu, X. F. Zhu, M. R. Li, R. P. O'Hayre and W. S. Yang, Nanoparticles at Grain Boundaries Inhibit the Phase Transformation of Perovskite Membrane, Nano Lett., 2015, 15(11), 7678-7683.

18 M. Adam, N. Gaponik, A. Eychmuller, T. Erdem, Z. SoranErdem and H. V. Demir, Colloidal Nanocrystals Embedded 
in Macrocrystals: Methods and Applications, J. Phys. Chem. Lett., 2016, 7(20), 4117-4123.

19 A. Benad, C. Guhrenz, C. Bauer, F. Eichler, M. Adam, C. Ziegler, N. Gaponik and A. Eychmuller, Cold Flow as Versatile Approach for Stable and Highly Luminescent Quantum Dot-Salt Composites, ACS Appl. Mater. Interfaces, 2016, 8(33), 21570-21575.

20 D. Vorontsov, S. Filonenko, A. Kanak, G. Okrepka and Y. Khalavka, Charge directed assembly of $\mathrm{CdTe} / \mathrm{CdS}$ nanoparticles inside monocrystalline $\mathrm{KH}_{2} \mathrm{PO}_{4}$, CrystEngComm, 2017, 19(45), 6804-6810.

21 Y. J. Liu, W. T. Yuan, Y. Shi, X. Q. Chen, Y. Wang, H. Z. Chen and H. Y. Li, Functionalizing Single Crystals: Incorporation of Nanoparticles Inside Gel-Grown Calcite Crystals, Angew. Chem., Int. Ed., 2014, 53(16), 4127-4131.

22 Y. J. Liu, H. D. Zang, L. Wang, W. F. Fu, W. T. Yuan, J. K. Wu, X. Y. Jin, J. S. Han, C. F. Wu, Y. Wang, H. L. L. Xing, H. Z. Chen and H. Y. Li, Nanoparticles Incorporated inside Single-Crystals: Enhanced Fluorescent Properties, Chem. Mater., 2016, 28(20), 7537-7543.

23 M. Calvaresi, G. Falini, L. Pasquini, M. Reggi, S. Fermani, G. C. Gazzadi, S. Frabboni and F. Zerbetto, Morphological and mechanical characterization of composite calcite/ SWCNT-COOH single crystals, Nanoscale, 2013, 5(15), 6944-6949.

24 Y.-Y. Kim, K. Ganesan, P. Yang, A. N. Kulak, S. Borukhin, S. Pechook, L. Ribeiro, R. Kröger, S. J. Eichhorn, S. P. Armes, B. Pokroy and F. C. Meldrum, An artificial biomineral formed by incorporation of copolymer micelles in calcite crystals, Nat. Mater., 2011, 10(11), 890-896.

25 Y. Y. Kim, M. Semsarilar, J. D. Carloni, K. R. Cho, A. N. Kulak, I. Polishchuk, C. T. Hendley, P. J. M. Smeets, L. A. Fielding, B. Pokroy, C. C. Tang, L. A. Estroff, S. P. Baker, S. P. Armes and F. C. Meldrum, Structure and Properties of Nanocomposites Formed by the Occlusion of Block Copolymer Worms and Vesicles Within Calcite Crystals, Adv. Funct. Mater., 2016, 26(9), 1382-1392.

26 A. N. Kulak, R. Grimes, Y. Y. Kim, M. Semsarilar, C. AnduixCanto, O. Cespedes, S. P. Armes and F. C. Meldrum, Polymer-Directed Assembly of Single Crystal Zinc Oxide/ Magnetite Nanocomposites under Atmospheric and Hydrothermal Conditions, Chem. Mater., 2016, 28(20), 7528-7536.

27 A. N. Kulak, P. C. Yang, Y. Y. Kim, S. P. Armes and F. C. Meldrum, Colouring crystals with inorganic nanoparticles, Chem. Commun., 2014, 50(1), 67-69.

28 Y. Ning, L. Fielding, J. Nutter, A. Kulak, F. Meldrum and S. Armes, Spatially-Controlled Occlusion of PolymerStabilized Gold Nanoparticles within ZnO, Angew. Chem., Int. Ed., 2019, 58(13), 4302-4307.

29 G. Lu, S. Li, Z. Guo, O. K. Farha, B. G. Hauser, X. Qi, Y. Wang, X. Wang, S. Han, X. Liu, J. S. DuChene, H. Zhang, Q. Zhang, X. Chen, J. Ma, S. C. J. Loo, W. D. Wei, Y. Yang, J. T. Hupp and F. Huo, Imparting functionality to a metal-organic framework material by controlled nanoparticle encapsulation, Nat. Chem., 2012, 4(4), 310-316.
30 J. H. Han, S. P. Luo, X. W. Yin, Y. Zhou, H. Nan, J. B. Li, X. Li, D. Oron, H. P. Shen and H. Lin, Hybrid PbS Quantum-Dot-inPerovskite for High-Efficiency Perovskite Solar Cell, Small, 2018, 14(31), 1801016.

31 Y.-C. Chien, D. L. Masica, J. J. Gray, S. Nguyen, H. Vali and M. D. McKee, Modulation of Calcium Oxalate Dihydrate Growth by Selective Crystal-face Binding of Phosphorylated Osteopontin and Polyaspartate Peptide Showing Occlusion by Sectoral (Compositional) Zoning, J. Biol. Chem., 2009, 284(35), 23491-23501.

32 B. Marzec, D. C. Green, M. A. Holden, A. S. Coté, J. Ihli, S. Khalid, A. Kulak, D. Walker, C. Tang, D. M. Duffy, Y.-Y. Kim and F. C. Meldrum, Amino Acid-Assisted Incorporation of Dye Molecules within Calcite Crystals, Angew. Chem., Int. Ed., 2018, 57(28), 8623-8628.

33 F. Nudelman, G. de With and N. Sommerdijk, Cryo-electron tomography: 3-dimensional imaging of soft matter, Soft Matter, 2011, 7(1), 17-24.

34 H. Y. Li, H. L. Xin, D. A. Muller and L. A. Estroff, Visualizing the 3D Internal Structure of Calcite Single Crystals Grown in Agarose Hydrogels, Science, 2009, 326(5957), 1244-1247.

35 M. Dierolf, A. Menzel, P. Thibault, P. Schneider, C. M. Kewish, R. Wepf, O. Bunk and F. Pfeiffer, Ptychographic X-ray computed tomography at the nanoscale, Nature, 2010, 467(7314), 436-439.

36 A. M. Wise, J. N. Weker, S. Kalirai, M. Farmand, D. A. Shapiro, F. Meirer and B. M. Weckhuysen, Nanoscale Chemical Imaging of an Individual Catalyst Particle with Soft X-ray Ptychography, ACS Catal., 2016, 6(4), 2178-2181.

37 J. Ihli, R. R. Jacob, M. Holler, M. Guizar-Sicairos, A. Diaz, J. C. da Silva, D. Ferreira Sanchez, F. Krumeich, D. Grolimund, M. Taddei, W. C. Cheng, Y. Shu, A. Menzel and J. A. van Bokhoven, A three-dimensional view of structural changes caused by deactivation of fluid catalytic cracking catalysts, Nat. Commun., 2017, 8(1), 809.

38 D. A. Shapiro, Y.-S. Yu, T. Tyliszczak, J. Cabana, R. Celestre, W. Chao, K. Kaznatcheev, A. L. D. Kilcoyne, F. Maia, S. Marchesini, Y. S. Meng, T. Warwick, L. L. Yang and H. A. Padmore, Chemical composition mapping with nanometre resolution by soft X-ray microscopy, Nat. Photonics, 2014, 8(10), 765-769.

39 J. Ihli, A. Diaz, Y. Shu, M. Guizar-Sicairos, M. Holler, K. Wakonig, M. Odstrcil, T. Li, F. Krumeich, E. A. Müller Gubler, W.-C. Cheng, J. A. van Bokhoven and A. Menzel, Resonant Ptychographic Tomography Facilitates ThreeDimensional Quantitative Colocalization of Catalyst Components and Chemical Elements, J. Phys. Chem. C, 2018, 122(40), 22920-22929.

40 Y. Ning, D. J. Whitaker, C. J. Mable, M. J. Derry, N. J. W. Penfold, A. N. Kulak, D. C. Green, F. C. Meldrum and S. P. Armes, Anionic block copolymer vesicles act as Trojan horses to enable efficient occlusion of guest species into host calcite crystals, Chem. Sci., 2018, 9(44), 8396-8401.

41 J. Ihli, P. Bots, A. N. Kulak, L. G. Benning and F. C. Meldrum, Elucidating Mechanisms of Diffusion-Based Calcium Carbonate Synthesis Leads to Controlled Mesocrystal Formation, Adv. Funct. Mater., 2013, 23(15), 1965-1973. 
42 M. Holler, J. Raabe, A. Diaz, M. Guizar-Sicairos, R. Wepf, M. Odstrcil, F. R. Shaik, V. Panneels, A. Menzel, B. Sarafimov, S. Maag, X. Wang, V. Thominet, H. Walther, T. Lachat, M. Vitins and O. Bunk, OMNY-A tOMography Nano crYo stage, Rev. Sci. Instrum., 2018, 89(4), 043706.

43 D. C. Green, J. Ihli, P. D. Thornton, M. A. Holden, B. Marzec, Y.-Y. Kim, A. N. Kulak, M. A. Levenstein, C. Tang, C. Lynch, S. E. D. Webb, C. J. Tynan and F. C. Meldrum, 3D visualization of additive occlusion and tunable fullspectrum fluorescence in calcite, Nat. Commun., 2016, 7, 13524.

44 J. Paquette and R. J. Reeder, New Type of Compositional Zoning in Calcite - Insights into Crystal Growth Mechanisms, Geology, 1990, 18(12), 1244-1247.

45 J. Paquette and R. J. Reeder, Relationship Between Surface Stucture, Growth Mechanism and Trace Element Incorporation in Calcite, Geochim. Cosmochim. Acta, 1995, 59(4), 735-749.

46 R. J. Reeder and J. Paquette, Sector Zoning in Natural and Synthetic Calcites, Sediment. Geol., 1989, 65(3-4), 239-247.

47 Y. Mei and M. Ballauff, Effect of counterions on the swelling of spherical polyelectrolyte brushes, Eur. Phys. J. E: Soft Matter Biol. Phys., 2005, 16(3), 341-349.

48 P. Pincus, Colloid Stabilization with Grafted Polyelectrolytes, Macromolecules, 1991, 24(10), 2912-2919.

49 R. Schweins and K. Huber, Collapse of sodium polyacrylate chains in calcium salt solutions, Eur. Phys. J. E: Soft Matter Biol. Phys., 2001, 5(1), 117-126.
50 Y. Ning, L. Han, M. Douverne, N. J. W. Penfold, M. J. Derry, F. C. Meldrum and S. P. Armes, What Dictates the Spatial Distribution of Nanoparticles within Calcite?, J. Am. Chem. Soc., 2019, 141(6), 2481-2489.

51 A. A. Chernov, Modern Crystallography III, in Springer Series in Solid State Sciences, Springer-Verlag, 1984, vol. 36.

52 K. R. Cho, Y. Y. Kim, P. C. Yang, W. Cai, H. H. Pan, A. N. Kulak, J. L. Lau, P. Kulshreshtha, S. P. Armes, F. C. Meldrum and J. J. De Yoreo, Direct observation of mineral-organic composite formation reveals occlusion mechanism, Nat. Commun., 2016, 7, 10187.

53 A. Brif, G. Ankonina, C. Drathen and B. Pokroy, Bio-Inspired Band Gap Engineering of Zinc Oxide by Intracrystalline Incorporation of Amino Acids, Adv. Mater., 2013, 26(3), 477-481.

54 S. Borukhin, L. Bloch, T. Radlauer, A. H. Hill, A. N. Fitch and B. Pokroy, Screening the Incorporation of Amino Acids into an Inorganic Crystalline Host: the Case of Calcite, Adv. Funct. Mater., 2012, 22(20), 4216-4224.

55 A. S. Schenk, I. Zlotnikov, B. Pokroy, N. Gierlinger, A. Masic, P. Zaslansky, A. N. Fitch, O. Paris, T. H. Metzger, H. Colfen, P. Fratzl and B. Aichmayer, Hierarchical Calcite Crystals with Occlusions of a Simple Polyelectrolyte Mimic Complex Biomineral Structures, Adv. Funct. Mater., 2012, 22(22), 4668-4676.

56 A. Berman, L. Addadi and S. Weiner, Interactions of Sea Urchin Skeleton Macromolecules with Growing Calcite Crystals - A Study of Intracrystalline Proteins, Nature, 1988, 331(6156), 546-548. 